\title{
PLAN DE ESTUDIOS PARA LA FORMACIÓN DEL CONTADOR PÚBLICO EN AMÉRICA LATINA
}

Dr. Julio Vicente Flores Konya*

\begin{abstract}
La Asociación Latinoamericana de Facultades y Escuelas de Contaduría (ALAFEC) cumpliendo el encargo del Consejo Ejecutivo Ampliado, realizada en México en el mes de marzo del 2002, presentó a la Asamblea General de ALAFEC, en la Ciudad de Mérida, Yucatán, México, realizado los días 12,13 y 14 de noviembre del 2003 entre otros, el tema central: "Las Bases para la Formación del Contador Público en América Latina».
\end{abstract}

Las Instituciones de Educación Superior están obligadas constantemente a replantear los temas educativos, respondiendo a las exigencias de los cambios en la estructura económica mundial, en concordancia con los avances de la Ciencia y la Tecnología, lo que permite lograr mejorar la formación de los recursos humanos, en este caso del Contador Público latinoamericano al servicio de cada país y vinculado internacional-mente, fortaleciendo la profesión ante los retos de las sociedades latinoamericanas en materia contable.

«El objetivo general es integrar por medio de una consulta regional, una propuesta minima para orientar los Planes de Estudio en Contaduria Pública que ofrecen las Instituciones de Educación Superior en América Latina".

A continuación se presenta el resultado del Tema Central de

\author{
ALAFEC: "LAS BASES PARA LA \\ CREACIÓN DE UN PLAN DE \\ ESTUDIOS PARA LA FORMACIÓN \\ DEL CONTADOR PÚBLICO EN \\ AMÉRICA LATINA".
}

\section{CONCLUSIONES}

Las propuestas de cada país se pueden integrar de manera estratégica considerando los siguientes puntos para la discusión y, en su caso, para la aprobación de las Bases de Creación de un Plan de Estudios Latinoamericano para la Contaduria Pública:

1. OBJETIVOS PROPUESTOS EN LA DEFINICIÓN DE LAS BASES PARA LA CREACIÓN DE UN PLAN DE ESTUDIOS LATINOAMERICANO PARA LA CONTADURÍA PÚBLICA

- Alcanzar una sólida base académica con un alto contenido teórico en todas las áreas de conocimiento definidas en la licenciatura para que permita la homologación del quehacer profesional una vez graduado el estudiante en cualquier país de Latinoamérica.

- Tener la suficiente flexibilidad para que cada país o región

- Docente Principal y Decano de la Facultad de Ciencias Contables 
pueda exponer en el Plan de Estudios sus propias caracteristicas o particularidades adecuadas a la realidad local.

- Integrar el sistema de conocimientos y habilidades adquiridos en el Plan de Estudios en el contexto nacional y latinoamericano.

\section{REgUISITOS MÍNIMOS DE LOS PLANES $Y$ PROGRAMAS DE ESTUDIO DE LA CONTADURÍA PÚBLICA}

Nuestro esfuerzo como académicos, se encamina a crear la metodología adecuada e innovadora para que la globalización y la tecnologia sirvan para el desarrollo armónico e integral de nuestros estudiantes.

Presentar y proponer información trascendental y decisiva sobre la situación y proyección financiera de entidades públicas, privadas $y$ sociales, que les permitan contribuir al desarrollo de las mismas.

Realizar ìnvestigaciones sobre las causas y las consecuencias de los diferentes fenómenos económicos para estar en posibilidad de prever contingencias y aminorar los riesgos financieros y ser factor de influencia en la actividad económica.

Desarrollar mecanismos para detectar y aprovechar a lo largo de su vida profesional nuevas oportunidades que se generan por la globalización en beneficio de los negocios y otro tipo de organizaciones.

Emplear eficazmente tecnología de información de carácter vanguardista.
Diseñar y desarrollar estrategias de autoaprendizaje e investigación que enriquezcan su formación competitiva y permitan aportar respuestas innovadoras a su actuación profesional.

Actuar con conciencia crítica y de manera ética y socialmente responsable en las actividades propias de su profesión y en su vida en general.

\section{ALGUNOS DE LOS OBJETIVOS PARTICULARES DE LAS BASES DE CREACIÓN DE LOS PLANES DE ESTUDIO}

Dirigir el proceso contable sobre la base de los principios internacionalmente reconocidos en la actividad económico-financiera, a través de las fases de sistematización, organización, valuación, procesamiento, evaluación e información que permitan su análisis y aporta los elementos de juicio necesarios para la toma de decisiones por parte de la dirección de una entidad dada, a partir de la política a seguir en este campo.

Aplicar técnicas y procedimientos actuales en la determinación y gestión de los costos, a través de su planeación, cálculo, análisis y control, que garanticen su utilización como instrumento de dirección.

Administrar los recursos financieros de una entidad, asi como sus relaciones con el resto de las organizaciones, empresas (públicas y privadas) económicas nacionales $\mathrm{e}$ internacionales de su entorno, mediante la utilización de las técnicas universalmente reconocidas $y$ aplicadas para la valuación, planeación, el análisis y el control. 
Proponer alternativas de financiamiento y utilización de los recursos, tanto para la actividad operativa como estratégica de una entidad, aplicando los procedimientos de la teoria financiera moderna y las técnicas actuales de la administración financiera, de forma tal que permita la toma de decisiones encaminadas al logro de una mayor eficiencia en la gestión de la organizaciōn económica de que se trate.

Aplicar técnicas y procedimientos de avanzada en la realización de auditorias, con programas establecidos o no a través de las fases de planeación, ejecución, información o dictamen, que determinen la racionalidad y veracidad de los resultados expuestos en los estados financieros, asi como evaluar cualquier otro elemento que permita orientar a la dirección para la toma de decisiones.

Proponer con independencia y creatividad las posibles soluciones a los distintos problemas de la profesión en búsqueda de la eficiencia económica sobre la base de los principios, normas y procedimientos del campo contable y financiero, aplicando la metodología de la investigación científica, utilizando adecuadamente la información bibliográfica, el idioma inglés y las técnicas de computacionales avanzadas.

\section{MARCO TEÓRICO DE LAS ÁREAS DEL CONOCIMIENTO}

Definición de las áreas de conocimiento. Para determinar las áreas del conocimiento básico se debe entender la diferencia entre disciplina y área de conocimiento:
Disciplina. Es un campo particular del conocimiento que ha logrado determinar tanto su objeto de estudio como las (otras) disciplinas que tangencialmente le ayudan a estudiar este objeto. Asimismo, tiene delimitadas las fronteras de su campo respecto de otras disciplinas que le sirven de sustento teórico y finalmente, también tiene determinadas las metodologias que mejor permiten su estudio.

Área de conocimiento. Es un campo teórico formado por un conjunto de núcleos problemáticos comunes que coadyuvan al análisis de objeto de una disciplina; asimismo, permite enfocar de una manera rigurosa y particular las relaciones conceptuales de los núcleos mencionados de manera que los cohesione y los articule en una totalidad que les otorgue un sentido de identidad.

Las consideraciones que se deberán hacer con respecto a este análisis antes de presentar las áreas de conocimiento definidas para la formación del profesional son:

1. Las áreas consideradas no son necesariamente las definitivas que puedan existir, ya que cualquier disciplina que se estudie cambia con el tiempo, y América Latina no es la excepción. De esta misma manera hay que considerar que las áreas del conocimiento hoy propuestas pueden modificarse según las necesidades que se presenten en los futuros ámbitos de desempeño profesional.

2. No puede pasarse por alto la existencia de varias ciencias o 
disciplinas que de una manera $\mathrm{u}$ otra complementan el conocimiento de estas áreas de conocimiento y auxilian en la comprensión de los fenómenos contables o administrativos, pero que no constituyen materia de estudio propia. En estos casos no se ha intentado definirlas ni justificarlas como áreas de estudio particular, ni tampoco incluyéndolas como materia de examen profesional o de grado. Se consideran como disciplinas complementarias a algunos aspectos de derecho, economia, matemáticas y ética.

\section{CRITERIOS DE JUSTIFICACIÓN DE CADA ÁREA DE CONOCIMIENTO}

Se ha establecido dentro de este trabajo cuatro criterios para justificar la particularización de un campo de conocimiento como un área académica especifica:

I. El grado de incidencia en la formación del carácter propio de una carrera.

II. El grado de complejidad de su estudio, que da pauta no solamente a la realización práctica de una serie de actividades, sino además a un contenido teórico profundo, como en el caso de administración y finanzas.

III. El grado de especificidad de su materia, que se forma especialmente por la relación que guarda con otras disciplinas no contables o administrativas, como son operaciones con matemáticas, auditoría, con estadística o contribuciones, con Derecho Fiscal, por citar sólo algunos ejemplos.

IV. La existencia de un cuerpo de conocimientos teóricos reconocido por los estudios de la disciplina de la cual forma parte.

Con base en los criterios antes mencionados, asi como la razón por la que se particularizó cada área, se proponen las siguientes ÁREAS DE CONOCIMIENTO.

\section{6. ÁREAS BÁSICAS DEL CONOCIMIENTO}

- Contabilidad

- Finanzas

- Costos

- Contraloría

- Impuestos

- Auditoría

La propuesta del contenido general de las áreas estructurales de la profesión será:

\subsection{Contabilidad}

Definición.- Es el área cuyo objetivo es el registro y sintesis de los efectos financieros de las operaciones que realiza una entidad económica, así como de los hechos económicos externos que le afectan, para informar a los interesados en tomar decisiones sobre las finanzas y el control financiero de esa entidad y facilitar, por lo tanto, esta área.

Razón de Particularización del Area.- Se considera, además de un 
subsistema organizacional, el área básica de la carrera de los profesionales de la Contaduría, ya que gran parte de su actuación está basada en la misma; también proporciona bases del conocimiento indispensables para el desarrollo de áreas más especializadas de la profesión como pueden ser auditoria, finanzas o contribuciones.

Resulta, asimismo importante como auxiliar, tanto para otras carreras de la Facultad como para algunos postgrados de la misma, por la información importante que proporciona para la toma de decisiones dentro de las organizaciones.

\subsection{Finanzas}

Definición.- Es el ārea en donde se estudian las técnicas y los procedimientos para planear, organizar y controlar la obtención y utilización óptimas de los recursos monetarios, provenientes de fuentes internas y externas de una organización, así como la estructura y funcionamiento de los mercados financieros, para lograr su mejor aprovechamiento en el cumplimiento de los objetivos de la organización.

Razón de Particularización del Área.- La razón primordial de la existencia de la contaduria y administración es la optimación de los recursos monetarios o equivalentes de una organización. Alrededor de este rubro se desarrollan las carreras e, inclusive, todas sus áreas tienen como objetivo contribuir de alguna manera a que las finanzas puedan ser manejadas eficientemente $y$, aunque su estudio es realizado en forma teórica principalmente por los profesionales de la contaduría, no puede soslayarse la importancia que reviste para la administración, lo que hace indispensable su estudio profundo y específico.

\subsection{Contraloria}

Definición.- Es el área cuyo objeto de estudio es la planeación financiera y el control de los recursos y verificación de las operaciones en la organización, para lograr el uso eficiente de los primeros y la eficacia en los segundos, a fin de que se alcancen los objetivos y metas establecidas en las mismas.

Razón de Particularización del Área.- La importancia que tiene el área dentro del desarrollo cotidiano de una organización, por la gran responsabilidad que conlleva, tanto en el plano financiero como sus repercusiones en el nivel de empleos y generación de riqueza, obligan a un estudio particularmente profundo y específico del área.

\subsection{Costos}

Definición.- Es el área cuyo objeto de estudio son los procesos enfocados a identificar, medir. acumular, analizar e interpretar los elementos del costo asociados con la producción y comercialización de bienes y servicios, con el propósito fundamental de tomar decisiones para alcanzar los objetivos de planeación estratégica establecidos previamente en la organización.

Razón de Particularización del Área.- No obstante que la contabilidad de costos es una rama 
determinada de la contabilidad, tradicionalmente ha sido estudiada por separado de la misma debido al grado de especialización que debe tener el profesional que se dedique a ella; también por su relación estrecha con disciplinas como la ingenieria -algunos aspectos de los sistemas de producción de bienes y servicios, así como la comprensión que se debe tener de los mismos se derivan de esta relación. Gran parte de su importancia radica en la información que genera para la toma de decisiones específicas en las áreas de transformación de una organización.

\subsection{Impuestos}

Definición.- Es el área en que se estudia la naturaleza de las tributaciones que debe enfrentar las personas físicas y morales para sufragar el gasto público que establece la legislación, incluyendo las normas, los métodos, las técnicas y los procedimientos necesarios para planear y controlar su cumplimiento y la propuesta de las mejores alternativas juridicas $y$ financieras tanto para el Estado como para los contribuyentes.

\section{Razón de Particularización del} Area.- El cumplimiento de las obligaciones fiscales y de la complejidad de los sistemas tributarios de nuestro país, fenómeno notable por la inestabilidad de la legislación correspondiente, exigen el constante estudio y actualización en la materia de los profesionales de nuestras carreras, lo que ha obligado a delimitar el área de contribuciones, conformándola como un campo de estudio específico.

\subsection{Auditoria}

Definición.- Es el área de conocimiento cuyo objetivo de estudio es el conjunto de técnicas que se aplican para evaluar el funcionamiento de la contabilidad y el control interno de una organización, con el fin de obtener evidencia suficiente y opinar sobre el estado que guardan estas funciones con relación a los principios de contabilidad, normas y procedimientos establecidos en la profesión contable, informando a los interesados mediante un dictamen que les permita tomar decisiones al respecto.

Razón de Particularización del Área.- La Contabilidad es, dentro de una organización, un sistema de información muy complejo y como tal requiere de un control que regule el funcionamiento de la misma, además de que se detecte las posibles desviaciones del objetivo que tiene, ya que los usuarios de la información por lo general no conocen directamente lo que aconteció en realidad y requieren, por tanto, de un profesional que les asegure que la información presentada es una declaración objetiva de los sucesos reales y de sus resultados. Este control es realizado por las diferentes fases de auditoria que cuenta con métodos propios y que, en consecuencia, tiene que ser estudiada como un área específica.

\section{7. ÁREAS COMPLEMENTARIAS DEL CONOCIMIENTO}

- Administración

- Informática Aplicada

- Matemática Avanzada y Estadística 
- Derecho

- Humanidades y Ciencias Sociales

- Economía

- Producción

- Mercadotecnia

- Seminario de Investigación

- Recursos Humanos

- Dirección y Operaciones

\section{PERFIL DEL EGRESADO LATINOAMERICANO}

El perfil del egresado deberá contar con conocimientos, valores y actitudes comunes a nivel latinoamericano los que son propuestos. Se encontraron convergencia entre todos los miembros de la Comisión de ALAFEC respecto a estos conocimientos, valores y actitudes, que se resumen en la siguiente tabla:

\begin{tabular}{|l|l|}
\hline \multicolumn{1}{|c|}{ CONOCIMIENTOS } & \multicolumn{1}{|c|}{ HABILIDADES Y ACTITUDES } \\
\hline Vinculación Teoría-Práctica & Formación Integral \\
Información Financiera & Habilidades Directivas y Administrativas \\
Fiscal & Trabajo en Equipo \\
Metodología de la Investigación & Ética \\
Control de Operaciones & Calidad \\
Auditoría & Seguridad en si mismo \\
Finanzas y Proyectos de Inversión & $\begin{array}{l}\text { Facilitar Procesos Contables y } \\
\text { Administrativos } \\
\text { Plantación Estratégica. }\end{array}$ \\
Reingeniería de Procesos. & Compromiso Social y Espiritu de Servicio \\
Humanísticas. & $\begin{array}{l}\text { Solución de Problemas y Tomas de } \\
\text { Decisiones } \\
\text { Creatividad y Espiritu Emprendedor } \\
\text { Diseñar, Implementar y Evaluar } \\
\text { Sistemas } \\
\text { Expresión Oral y Escrita }\end{array}$ \\
\hline
\end{tabular}




\section{GRANDES CAMPOS DE ACTUACIÓN PROFESIONAL DEL CONTADOR PÚBLICO}

La preparación y formación del egresado en Contaduría Pública, debe estar dirigida a garantizar su certera actuación como profesional en las actividades de cualquier sector de la economía nacional de que se trate y de su inserción dentro de la economía internacional.

Especial atención deberá prestar cada pais a las prioridades que caracterizan la economia nacional, aun cuando existen sectores que son comunes en general: comercio, industria, agricultura, construcción, transporte, entre otros. En muchos paises de Latinoamérica y en especial, en el Caribe, el turismo juega un papel específico que no debe ser ignorado.

El profesional graduado deberá estar preparado también para actuar en los sectores no productivos que por su importancia $\mathrm{y} / \mathrm{o}$ alcance demandan una considerable cantidad de recursos financieros, materiales y humanos para su gestión, entre los que cabe destacar: la salud pública, la educación, la asistencia social, la cultura, el arte, la ciencia y la técnica, la administración pública, entre otros.

El actual desarrollo de las instituciones financieras bancarias y bursátiles, así como el papel activo que las mismas desempeñan en las economias nacionales $\mathrm{e}$ internacionales, se constituyen en una esfera de esencial importancia para la formación de este profesional.

\section{EL POSTGRADO EN CONTADURÍA}

Para mejorar el proceso de educación de América Latina, es necesario atender el Postgrado, debido a que éste será el camino para desarrollar la profesión y llevarla a niveles de excelencia, ya sea en la formación de profesores o en la formación de investigadores.

Las oportunidades son las siguientes:

- Un mercado creciente: en el caso de México se puede observar en las estadísticas un resultado interesante sobre los estudiantes del Postgrado. La Maestría va creciendo con velocidad, ya que en 1984 eran más o menos 12000 alumnos, y ahora estamos hablando de casi 37000 alumnos; con el crecimiento y la demanda de servicios de postgrado, la tendencia indica que nuestras escuelas no tendrán problemas para tener alumnos de Postgrado. Esto puede ser analizado en forma análoga en Latinoamérica.

El Doctorado es donde la tendencia no es tan rápida; por ello, las universidades tenemos que dedicar más tiempo y esfuerzo a ello; ese es el reto de largo plazo en las universidades latinoamericanas.

- Las escuelas con Postgrado en Negocios deben visualizar la educación en términos de ciencia, arte y acción; o sea se tiene que reforzar esa parte del proceso reflexivo de las personas y los alumnos; la parte de creatividad, de propuestas y el enfoque al desarrollo sustentable. 
- También se debe buscar fortalecer y actualizar los programas de postgrado, buscar que sean relevantes y útiles. Implementar áreas nuevas como Doctorados en Contaduria, lo cual sería un reto interesante.

- Elevar el nivel de exigencia y calidad, tratando de ser mejores; para ello tenemos que ver quiénes son los mejores y tratar de ser igual que ellos.

Ahora vamos a ver una segunda lista de oportunidades:

- Un área de oportunidad es buscar alianzas estratégicas entre instituciones afiliadas.

- Uso inteligente del talento: partiendo del hecho de que las universidades no estamos formando el suficiente número de doctores en las diversas áreas del conocimiento de nuestra profesión.

- Aumentar el porcentaje de profesores de planta a tiempo completo para que se dediquen a ser investigadores y docentes de tiempo completo.

- Contar con profesores comprometidos para orientar en habilidades, actitudes y valores que sean un ejemplo.

- Profesores que tengan un grado superior al nivel en que imparten cátedra es importante para la obtención de la acreditación de los programas académicos en que las universidades estamos inmersas en el sentido de desarrollo académico permanente.

- Firmar convenios que permitan explorar alternativas de cómo entrenar a los profesores, con una variedad de métodos de enseñanza y recursos que ayuden a los estudiantes a adquirir mejores conocimientos.

Se sabe de la importancia que tiene para cada país el formar gente con nivel de Postgrado en Contaduria y no tienen un nicho dentro del desarrollo; entonces éste es uno de los retos que hay que resolver en el corto plazo con el establecimiento de un sistema de reconocimiento de los postgrados.

\section{INVESTIGACIÓN}

Reconociendo como supuesto básico la importancia del desarrollo de la investigación a nivel latinoamericano y la falta de investigadores, se proponen las siguientes estrategias para fortalecer la investigación en contaduría.

\subsection{Estrategias Propuestas para la Investigación}

Formar una base de datos de todas las universidades afiliadas a ALAFEC, de los investigadores, líneas y proyectos de investigación, además de las necesidades para su desarrollo.

Conocer las líneas de investigación requiere de las instituciones para promover el intercambio de investigadores.

Desarrollar periódicamente un evento internacional relativo a los avances de investigación que se tengan en los diversos países de América Latina.

Proyectos de vinculación con los sectores productivo, gubernamental 
y social, para la realización de investigaciones de interés mutuo, para desarrollo de cadenas productivas, mejoras en los procesos de gestión o estudios de coyuntura.

Promover la formación doctoral de la plantilla docente actual, para que desarrollen investigación dentro de su función académica.

Repatriar a doctores que estuviesen en el extranjero, para promover con ello el generar cuadros de alto nivel en investigación.

Generar un proyecto para definir una convocatoria que promueva el concurso de las áreas fundamentales de la Contaduria.

\section{RECOMENDACIONES}

En caso de la Universidad Nacional Mayor de San Marcos, existen coincidencias en su mayor parte en los Planes de Estudios. Sin embargo, debemos considerar algunos aspectos importantes, entre otros los siguientes:
1. La base de la enseñanza en períodos semestrales.

2. Confirmar la duración de los estudios profesionales en cinco años, conciliando la teoría y la práctica.

3. Fortalecer la profesión a largo plazo, formando maestros $y$ doctores encargados de formar los futuros académicos de América Latina.

4. Desarrollar, sumando esfuerzos, la investigación, participando todos los países de América Latina.

5. Tomar como áreas básicas del conocimiento las señaladas en el punto 6: Áreas Básicas del Conocimiento, y en el punto 7: Áreas Complementarias del Conocimiento.

6. El contador público debe tener una formación básicamente con sentido ético, cuyos servicios profesionales respondan ejemplarmente a la sociedad. 